\title{
Application of Proteases as Molecular Imaging Tools for Early Gastric Cancer Detection: In Vitro and Ex Vivo Investigation
}

Wajana Lako Labisso ${ }^{* *}$ and Daniel Seifu²

${ }^{1}$ Department of Pathology, School of Medicine, College of Health Sciences, Addis Ababa University, Ethiopia

${ }^{2}$ Department of Medical Biochemistry, School of Medicine, College of Health Sciences, Addis Ababa University, Ethiopia

\begin{abstract}
Background: Substantial miss-rate of gastric cancer by conventianl endoscope was reported, indicating the importance of novel biomarkers for early detection of precancerous and cancerous lesions.

Objective: To identify potential biomarker molecules for early gastric cancer detection and evaluation of their application for molecular in vivo imaging.

Method: Besides the CEA-TAG+/- mouse model, 3 human and 2 murine gastric cancer cell lines were applied in this study. Cells lines were cultured at standard conditions. To determine the expression pattern of the targets, total RNA was extracted from cultured cells; cDNA was synthesised and qPCR was performed using spcefic primers. The murine stomach was removed and washed with PBS. The stomach was macroscopically categorized into tumor region and normal tissues. Histopathology and imunohistochemitry was performed in one half of the tumor tissues using specific antibodies. In the other half of the tumour tissue, RNA was extracted, reverse transcribed into cDNA and QPCR was performed. Histopathology, immhunohistochemistry and GPCR was performed for tumor-adjescent normal tissues to compare the difference in expression pattern of the target. Finally, a cathepsin activatable probe was injected into mice intravenously. Ex vivo fluorescent imaging was performed after 24 hours of intravenous injection of a cathepsin activatable probe. Gene expression was quantified by standard method with normalizing CT values to the housekeeping gene (Cyclophilin $A)$.

Results: Significantly elevated expression of cathepsins B, H and MMP2 in human and murine gastric cancer cell lines was detected. In addition, increased expression of cathepsins and MMPs was observed in murine primary gastric tumour specimens compared to the corresponding adjacent normal gastric mucosa $(p<0.05$, tumor versus normal mucosa). Accordingly, the NIRF probe was specifically activated in stomach tumor of CEA-TAG+/- mice and allowed ex vivo detection of the stomach tumor by Odyssey planar near-infrared scanner. Furthermore, immunohistochemical stainings with antibodies specific for cathepsins and MMPs indicated a stronger signal towards the tumor tissues compared to the adjacent normal mucosa.

Conclusion: Cathepsins and MMPs are potential biomarkers for detection of high grade dysplasia and early gastric cancer in mouse models, revealing the potential applicability of the biomarkers in tumor imaging and therapeutic monitoring.
\end{abstract}

Keywords: Proteases; Cathpsins; MMPs; Biomarkers; Gastric cancer; Imaging

\section{Introduction}

A critical step in improving the outcome of gastric cancer patients is to detect gastric lesions at their premalignant stages, thereby permitting minimal invasive therapy $[1,2]$. Thus far, high grade dysplasia and early gastric cancers are often missed during conventional endoscopic examination of the upper gastrointestinal tract [3]. Therefore, the development of highly sensitive and specific techniques for preneoplastic lesions and early gastric cancer detection is extremely important. Recent progress in imaging research indicated that molecular imaging in animal models is possible using antibodies or ligands, labeled with radioactives or flourophores [4,5]. In addition, activable near infrared fluorescence (NIRF) imaging probes ("smart probes") for preneoplastic and neoplastic lesions have been shown to detect adenomas in the APCmin/+ mouse models. These "smart probes" are specifically activated by tumor specific proteases. Even lesions smaller than $500 \mu \mathrm{m}$ that were macroscopically invisible were detected with the aid of smart probes, indicating that these probes can be used for early detection of adenomas [6-9] Proteases are heterogeneous group of enzymes that play a pivotal role in many pathophysiologic conditions, including gastric carcinogenesis. Among others matrix metalloproteinases (MMPs) and cathepsins are well known for their crucial application in cancer diagnosis and treatment [10-12]. They have the capacity to completely remodel the extracellular matrix $(\mathrm{ECM})$ and can therefore change several biologic processes, including angiogenesis, growth factor bioavailability, cytokine modulation, receptor shedding, metastasis, proliferation, invasion, and apoptosis [10-12]. Proteases and their protein inhibitors have been implicated in several other forms of cancers too [11-13]. In addition, several extracellular proteases have been shown to alter tumor aggressiveness and patient response to therapy [14-16]. Elevated expression of the proteases at early stage of carcinogenesis also gives an opportunity to make use of them for early detection of tumor $[17,18]$. Therefore, the

*Corresponding author: Wajana Lako Labisso, Department of Pathology, Schoo of Medicine, College of Health Sciences, Addis Ababa University, P.O. Box, 9086 Addis Ababa, Ethiopia, Tel: +25191 142 9050; E-mail: wajana.lako@aau.edu.et

Received November 24, 2017; Accepted November 28, 2017; Published November 30, 2017

Citation: Labisso WL, Seifu D (2017) Application of Proteases as Molecular Imaging Tools for Early Gastric Cancer Detection: In Vitro and Ex Vivo Investigation. J Mol Biomark Diagn 8: 366. doi: 10.4172/2155-9929.1000366

Copyright: (c) 2017 Labisso WL, et al. This is an open-access article distributed under the terms of the Creative Commons Attribution License, which permits unrestricted use, distribution, and reproduction in any medium, provided the original author and source are credited. 
objective of the current study was to explore cathepsins and MMPs as potential biomarker molecules for early gastric cancer detection and evaluation of their application for molecular in vivo imaging. Herein, we report that proteases display a biomarker role and enable early detection before metastatic spread.

\section{Materials and Methods}

\section{Cell culture conditions and preservation of cells}

Generally, 3 human and 2 murine gastric cancer cell lines were applied in this study (Table 1). All of the cell lines were cultured at $37^{\circ} \mathrm{C}$ and $5 \% \mathrm{CO}_{2}$, regardless of their origin of generation. The required media composition for all human origins was $500 \mathrm{ml}$ DMEM media (Invitrogen, Carlsbad, CA), 10\% FCS and 1\% P/S. We applied $500 \mathrm{ml}$ DMEM media, $10 \%$ FCS, $1 \%$ P/S, $1 \%$ NEAA and $1 \%$ sodium pyruvate for the murine gastric cancer cell lines. To subculture, cells were washed with sterile PBS in a laminar flow hood, trypsinated for 1-5 min at $37^{\circ} \mathrm{C}$ and passaged in a new flask containing fresh medium. For cryopreservation, the trypsinated cells were suspended in fresh medium and centrifuged at $1000 \mathrm{rpm}$ for $5 \mathrm{~min}$. The supernatant was discarded carefully. Finally, the pellet was re-suspended in ice-cold freezing medium and kept in liquid nitrogen for further use.

\section{Total RNA isolation and preparation of cDNA library for $\mathrm{qPCR}$}

Cells were grown to $80 \%$ confluency, washed with PBS and lysed with RLT buffer containing ß-mercaptoethanol (Sigma-Aldrich Chemie $\mathrm{GmbH}$, Steinheim). Then, the cells were collected into a BioPur safelock reaction tube. Total RNA from samples in RLT buffer was isolated with RNeasy Kit (Invitrogene) and treated with DNAse I (Qiagen GmbH, Hilden) according to manufacturer's protocol. A single $\mu \mathrm{g}$ RNA per $50 \mu \mathrm{l}$ mix were reversely transcribed using random hexamer primers, TaqMan reverse transcription reagents and SuperScript II reverse transcriptase (Applied Biosystems Inc., Foster City, CA, USA). Finally, the reversely transcribed cDNA was stored at $-20^{\circ} \mathrm{C}$ for further use. In similar fashion, murine stomach was extracted, washed with PBS and macroscopically categorized into tumor region (most probably the antrum region in this case) and normal tissues (fundus/corpus) (Figure 1).

Twenty milligrams of each of the categorized tissues is minced into smaller pieces, and homogenized in RLT buffer. Homogenized tissues were either stored at $-80^{\circ} \mathrm{C}$ for further application or sonified in a silent crusher for RNA extraction. The extracted RNA was immediately reverse transcribed into $\mathrm{CDNA}$ and stored at $-20^{\circ} \mathrm{C}$ for further use.

\section{Quantitative polymerase chain reaction ( $\mathrm{qPCR}$ )}

Based on the manufacturer's guidelines, $\mathrm{qPCR}$ primers were designed using Primer Express software (Applied Biosystems). SYBR ${ }^{\circ}$ Green PCR Master Mix (Qiagen GmbH, Hilden )and $300 \mathrm{nM}$ of each primer were used to perform qPCR. Cyclophilin was used as a housekeeping gene for normalization of the samples. All samples were done in triplicates for statistical analysis. The primers used in this study were described in Tables 2 and 3.

Gene expression was quantified by standard method and CT values

\begin{tabular}{|c|c|}
\hline Cell line & Source \\
\hline KATO-III & American type culture collection (ATCC) \\
\hline AGS & Deutsche Sammlung von Mikroorganismen und Zellkulturen \\
\hline MKN45 & Kind gift from Dr. R.Mejías-Luque \\
\hline MGC4 & Kind gift from Dr. W. Zimmermann \\
\hline MGC8 & Kind gift from Dr. W. Zimmermann \\
\hline & Table 1: Cell lines used in this study. \\
\hline
\end{tabular}

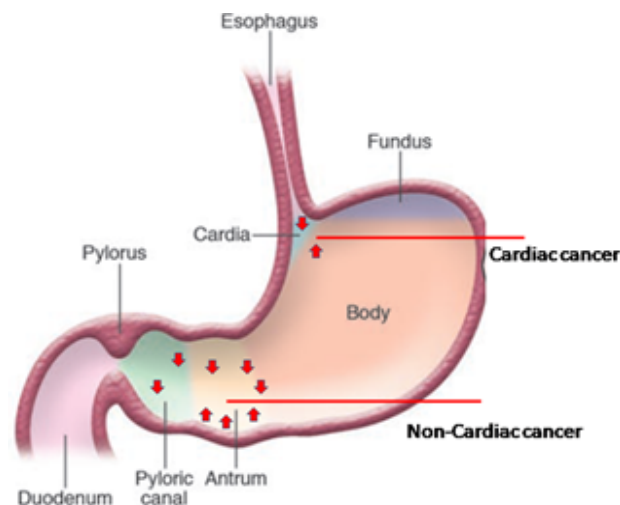

Figure 1: Anatomical sites of gastric cancer.

\begin{tabular}{|c|c|c|}
\hline Target name & Primer name & Primer sequence $\left(5^{\prime}-3^{\prime}\right)$ \\
\hline \multirow{2}{*}{ PPIA } & Cyc-FW & ATGGTCAACCCCACCGTGT \\
\hline & Cyc-RV & TCTGCTGTCTTTGGGACCTTGTC \\
\hline \multirow{2}{*}{ MMP9 } & MMP9-FW & GCACCACCACAACATCACCTAT \\
\hline & MMP9-RV & TGTACACGCGAGTGAAGGTGAG \\
\hline \multirow{2}{*}{ MMP13 } & MMP13-FW & TCCCAGTGGTGGTGATGAAGA \\
\hline & MMP13-RV & GGATTCCCGCGAGATTTGTAG \\
\hline \multirow{2}{*}{ MMP2 } & MMP2 -FW & CCTGAGATCTGCAAACAGGACAT \\
\hline & MMP2-RV & GCCAAATGAACCGGTCCTT \\
\hline \multirow{2}{*}{ MMP3 } & MMP3-FW & ACCTGGAAATGTTTTGGCCCAATGC \\
\hline & MMP3-RV & GGTCCCTGTTGTATCCTTTGT \\
\hline \multirow{2}{*}{ CTSB } & CTSB- FW & CTGTCGGATGAGCTGGTCAACT \\
\hline & CTSB -RV & CCACCCAGGAAGGTACCACATA \\
\hline \multirow{2}{*}{ CTSL } & CTSL-FW & CTGTTTTATGAGGCCCCCAGA \\
\hline & CTSL-RV & GCCCAACAAGAACCACACTGAC \\
\hline \multirow{2}{*}{ CTSS } & CTSS-FW & TCGACTCAGACGCTTCCTATCC \\
\hline & CTSS-RV & TGAACATGTGGCAGCACGAT \\
\hline \multirow{2}{*}{ CTS H } & CTS H_FW & TGGTTATTGCAAGTTCCAACC \\
\hline & CTS H-RV & GTGACTCAGGACTTCATGATG \\
\hline
\end{tabular}

Table 2: Human primer sets applied in $\mathrm{GPCR}$.

\begin{tabular}{|c|c|c|}
\hline Target name & Primer name & Sequence $\mathbf{( 5}^{\prime} \mathbf{-} \mathbf{3}$ ') \\
\hline \multirow{2}{*}{ MMP2 } & MMP2 -FW & ACACTGGGACCTGTCACTCC \\
\cline { 2 - 3 } & MMP2-RV & TGTCACTGTCCGCCAAATAA \\
\hline \multirow{2}{*}{ MMP13 } & MMP13-FW & TGGACCTTCTGGTCTTCTGG \\
\cline { 2 - 3 } & MMP13-RV & CTCATGGGCAGCAACAATAA \\
\hline \multirow{2}{*}{ CTSB } & CTSB- FW & GGAGATACTCCCAGGTGCAA \\
\cline { 2 - 3 } & CTSB -RV & CTGCCATGATCTCCTTCACA \\
\hline \multirow{2}{*}{ CTSL } & CTSL-FW & TCAGTGAGATCAGTTTGCCG \\
\cline { 2 - 3 } & CTSL-RV & TCCCTCAGTGCTCAGAACCT \\
\hline \multirow{2}{*}{ CTS H } & CTS H_FW & CCAGTGGGAAAATGCTGTCT \\
\cline { 2 - 3 } & CTS H-RV & TCCATGATGCCCTTGTTGTA \\
\hline \multirow{2}{*}{ PPIA } & Cyclo-FW & ATGGTCAACCCCACCGTGT \\
\cline { 2 - 3 } & Cyclo-RV & TTCTTGCTGTCTTTGGAACTTTGTC \\
\hline
\end{tabular}

Table 3: Murine primer sets applied in qPCR.

of qPCR were normalized to the housekeeping gene-cyclophilin A. After amplification, melting curve analysis was performed to ensure the specificity of the products. To ensure experimental accuracy, all reactions were performed in triplicate. Primers were tested for efficiency before applications.

\section{Murine ex-vivo imaging}

The CEA-TAG mouse model was kindly provided by Prof. 
Zimmermann [19] and applied in this study for imaging gastric cancer with the aid of "smart" probes. The expression pattern of cathepsins and matrix metalloprotinases (MMPs) as potential biomarker molecules for preneoplastic lesions and early gastric cancer detection was determined in the stomach tissues of these mice. For ex-vivo imaging of gastric cancer in these mice, $150 \mu \mathrm{l}$ prosense-750 (Perkinlmer, Inc. Boston, USA) probe was injected intravenously (IV) and the mice were kept back to their cage for 24 hours. After 24 hrs of injection, the mice were sacrified and the stomach was carefully removed. The stomach contents were washed with PBS and near-infrared fluorescent (NIRF) imaging was performed on a planar Odyssey scanner (LI-COR Bioscience Corporate, Lincoln, NE, USA) at $800 \mathrm{~nm}$. Afterwards, exvivo histological analysis was performed. All animal studies conducted meet the requirements of the European guidelines for the care and use of laboratory animals and were approved by the local authorities.

\section{Tissue sectioning and Haematoxylin and eosin (HE) staining}

Stomach tissue was washed with PBS, fixed in Roti ${ }^{\circledR}$ Histofix for 24 hrs, dehydrated using ASP300 Tissue Processor (Leica) and embedded in paraffin. A series of $4 \mu \mathrm{m}$ thick sections were cut for staining. Wax was removed from paraffin embedded tissue sections with the aid of $2 \mathrm{x}$ Roti ${ }^{\circledast}$ Histol (Carl Roth) for 5 minutes. Then, the tissue was rehydrated with the decreasing alcohol series (2x 100\%, 2x 96\% and 2x 80\% EtOH), each for 3 minutes. Sections were then stained in haematoxylin for 5 $\mathrm{sec}$, gently washed in tap water for $10 \mathrm{~min}$ and then stained in eosin for approximately $20 \mathrm{sec}$. Tissue was dehydrated with an increasing alcohol series (2x 80\%, 2x 96\% and 2x 100\% ethanol). After immersing 2x for 5 min in Roti ${ }^{\oplus}$ Histol (Carl Roth) slides were mounted in Pertex for microscopic observation.

\section{Immunohistochemistry}

The stomach tissue was rehydrated after de-paraffinizing the embedded stomach tissue sections. Microwave antigen retrieval was performed using unmasking solution (Vector Labs) for $9 \mathrm{~min}$. After a cooling period of at least $15 \mathrm{~min}$, slides were washed in distilled $\mathrm{H}_{2} \mathrm{O}$. Endogenous peroxidase reactivity was blocked by incubating slides in $3 \% \mathrm{H}_{2} \mathrm{O}_{2}$ for $20 \mathrm{~min}$. After washing with water and PBS for one and two times, respectively, incubation with $5 \%$ serum in PBS for $1 \mathrm{~h}$ was carried out to block unspecific antibody binding. Primary antibodies (Research \& Diagnostics Systems, Inc. (R\&D Systems), USA) were diluted to desired concentration in 3\% serum in PBS and incubated. In all cases, the primary antibody was diluted to a factor of 1:500, goat serum was used for blocking and incubation was for 1 $\mathrm{h}$ at room temperature (RT). The slides were washed three times with PBS to remove unspecifically bound primary antibody. Biotinylated secondary antibody was diluted (1:200) and incubated for $1 \mathrm{~h}$ at room temperature. After washing, VECTASTAIN ${ }^{\bullet}$ Elite ABC solution (Vector Labs) was added and afterwards slides were incubated with 3, 3'-diaminobenzidine tetrahydrochloride (DAB, Vector Labs) until suitable brown staining is developed. Slides were finally counterstained with hematoxylin and mounted in pertex.

\section{Results}

\section{Proteases are highly expressed in gastric cancer cell lines and tissues}

Cathepsins and matrix metalloprotinases (MMPs) are involved in tumor initiation, extracellular matrix degradation [10-13] and metastasis [14]. These enzymes are considered to be promising tools for in-vivo molecular detection of precancerous and cancerous lesions in several cancer types $[5,19,20]$. To extend further the application of these proteases for molecular detection of precursor and cancerous lesions in gastric cancer, their expression pattern in murine gastric cancer cell lines and stomach tissues was determined by qPCR. In the same fashion, the expression pattern of these proteases in human gastric cancer cell lines was detected. The results indicated that cathepsin B, Cathepsin $\mathrm{H}$ and MMP2 are overexpressed in KatoIII, AGS and HSC45-M2 cell lines. Only marginal expression of other cathepsins such as cathepsin $\mathrm{S}$ and L and MMPs such as MMP3, MMP9 and MMP13 was observed in human gastric cancer cell lines (Figures $2 \mathrm{~A}$ and $2 \mathrm{~B}$ ).

Analogous to its human counter parts, strong expression of cathepsin B was observed in murine gastric cancer cell lines (Figure $3 \mathrm{~A}$ ). Even stronger expression of cathepsin L was observed in murine gastric cancer cells than human gastric cancer cell lines. MMP2 expression level showed a high standard deviation in murine cell lines, indicating higher tumor heterogeneity in mice (Figure 3B).

It is noteworthy to mention that the expression pattern of cathepsins in human gastric cancer cell lines has association with that of murine ones. This was the reasons to investigate the expression patterns of relevant cathepsins and MMPs in a murine gastric cancer model. To this end, RNA was extracted from cancerous and adjacent normal mucosa of CEA-TAG mice and qPCR was performed for both cathepsins and MMPs. As expected, elevated expression of cathepsins (B, H and L) and MMP2 was observed from cancerous tissues of stomach of CEA-TAG mice. Interestingly, only marginal expression was observed in the adjacent normal mucosa (Figures 3C-3G).

To validate the expression of cathepsins and to examine the specify of the corresponding proteins to tumor tissues, immunohistochemistry was performed. In contrast to other cathepsins, stronger staining for cathepsins $\mathrm{B}$ was observed in tumor region of the stomach of $C E A$ $T A G$ mice. Very weak signal of all proteases was observed from the adjacent normal mucosa (Figures 4A and 4B). This is in agreement with qPCR results described in Figure 3. Similarly, stronger signal for cathepsins $\mathrm{H}$ and L, MMP2 and MMP3 were observed in tumor tissues than the adjacent normal mucosa (Figures $4 \mathrm{C}-4 \mathrm{~F}$ ).

In the same fashion, immunohistochemistry for representative MMPs was performed in murine stomach tissues. Higher expression of MMP2 and MMP3 was detected in cancerous tissues than the adjacent normal mucosa (Figures 4G-4J) whereas no significant expressions of other MMPs except MMP2 and 3 were detected in gastric cancer specimens.
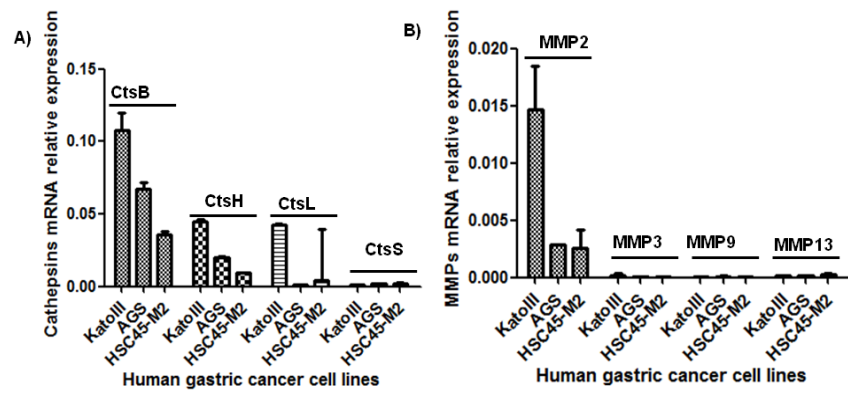

Figures 2: Depicted is mRNA expression of cathepsin (Cts) and matrix metalloproteinases (MMPs) in human gastric cancer cell lines. Human gastric cancer cell lines were grown to exponential phase and total RNA was isolated, reverse transcribed and $\mathrm{qPCR}$ was performed to determine the level of expression of mRNAs of (A) cathepsins and (B) MMPs. Cyclophilin was used as a house keeping gene. Experiments were done in triplicates and data are presented as mean and standard error of the mean (S.E.M). 
Citation: Labisso WL, Seifu D (2017) Application of Proteases as Molecular Imaging Tools for Early Gastric Cancer Detection: In Vitro and Ex Vivo Investigation. J Mol Biomark Diagn 8: 366. doi: 10.4172/2155-9929.1000366

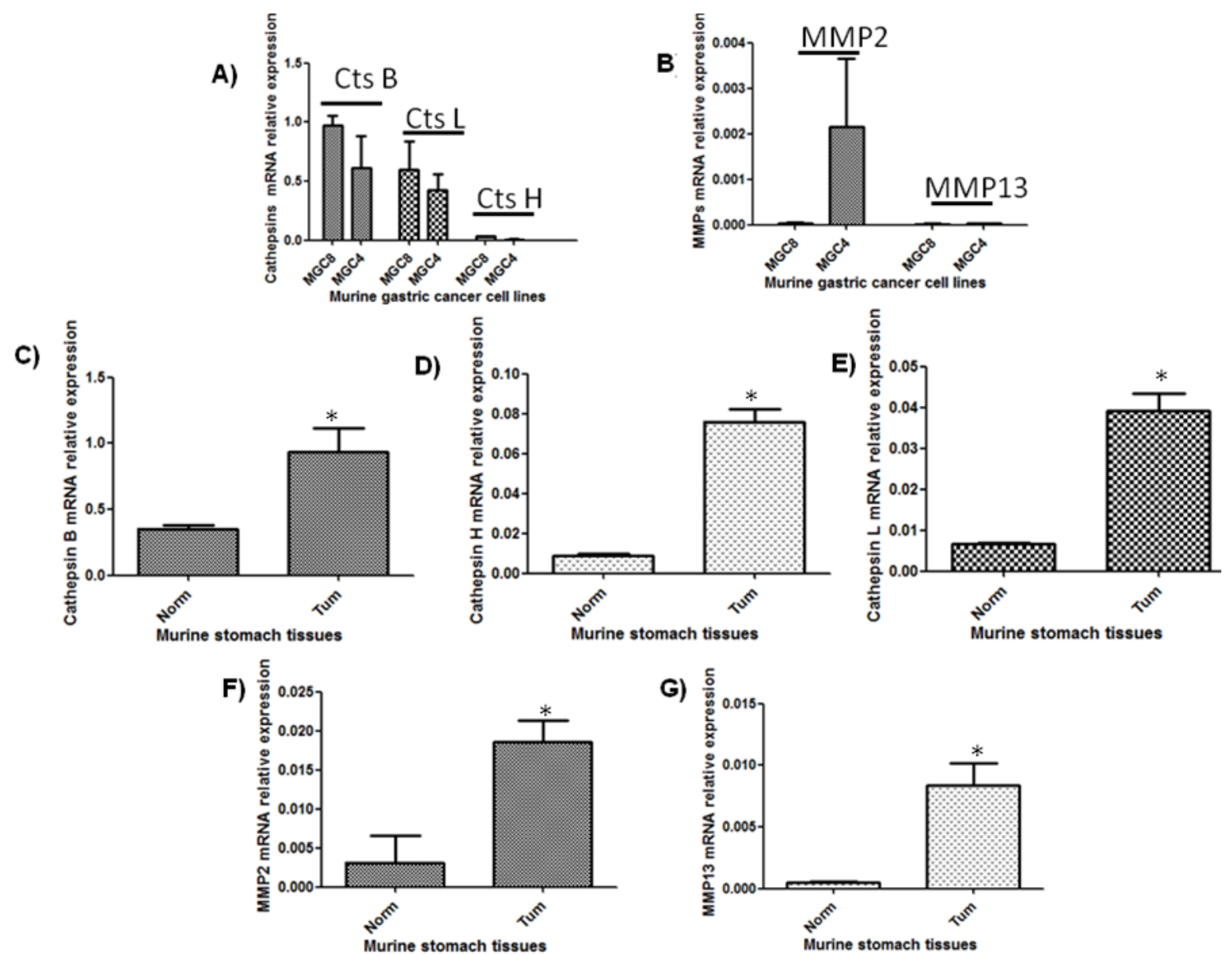

Figures 3: mRNA expression of cathepsin (Cts) and matrix metalloproteinases (MMPs) in murine gastric cancer cell lines and tissues. Murine gastric cancer cell lines were grown to exponential phase and total RNA was isolated, reverse transcribed and qPCR was carried out to determine the level of mRNA expression of cathepsins (Cts) (A) and matrix metaloprotinases (MMPs) (B). mRNA expression of cathepsin (Cts) and matrix metalloproteinases (MMPs) in murine stomach tissues was determined (C-G): Stomach from CEA-TAG mice was opened and washed with PBS and total RNA was isolated. Reverse transcription and qPCR was performed to determine level of expression of Cts (C-E) and MMPs (F-G) in cancerous tissues and normal mucosa. In all experiment, cyclophilin was used as a house keeping gene. At least three mice were included in all experiments. All experiments were done in triplicates and data are presented as mean and standard error of the mean (S.E.M). Student's two tailed t-test: * $\mathrm{p}<0.05$ (Tumor versus normal mucosa) value was considered as statistically significant.

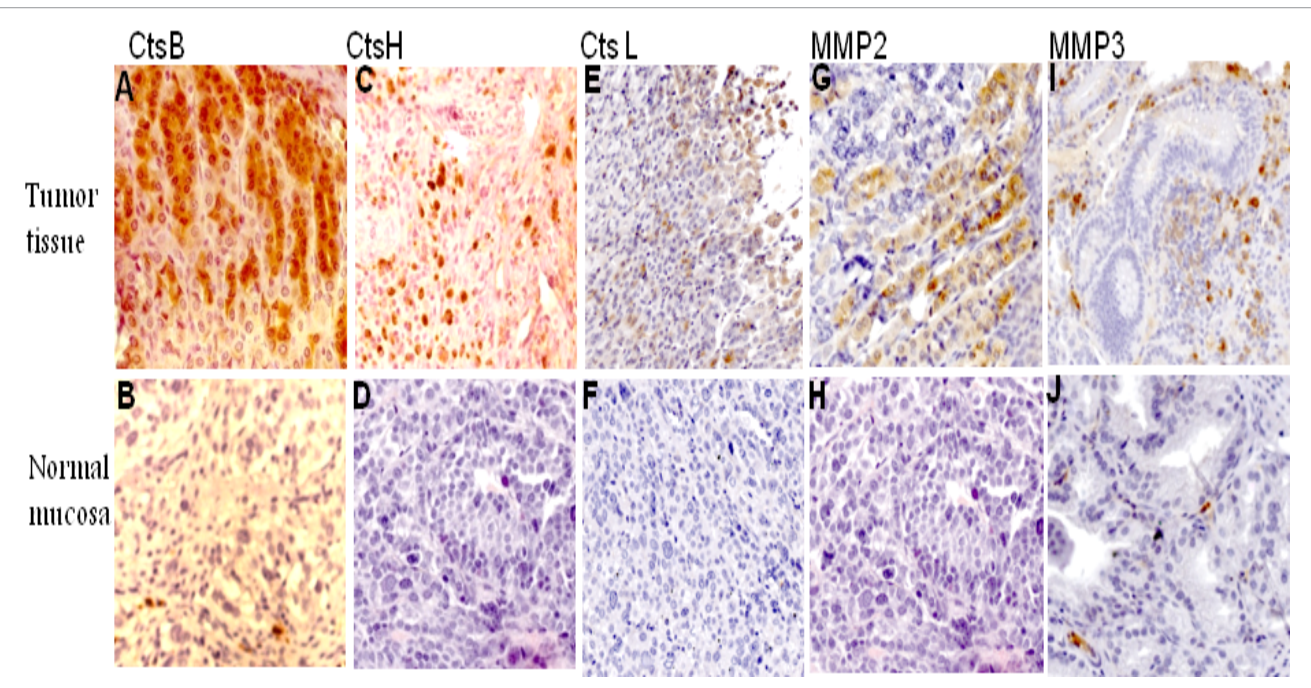

Figures 4: Representative results of immunohistochemistry of cathepsins (Cts) and matrix metalloprotinases (MMPs) in murine gastric cancer and normal adjacent mucosa. Stomach tissue from CEA-TAG mice was stained with antibodies specific for cathepsins B, H and L and matrix metalloprotinases MMP2 and MMP3. The upper row is from cancer tissues whereas the lower row is from adjacent normal mucosa. 


\section{Prosense-750 injected\#13260}
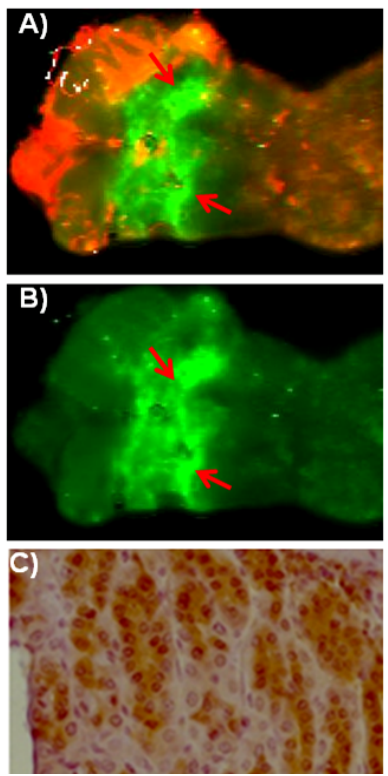

Figures 5: Representative pictures NIRF images. NIRF imaging of stomach tissues from CEA-TAG mice that bear stomach tumor indicates strong signa towards the antrum (A and $B$ ). Mice not injected with the probe served as controls ( $D$ and $E)$. Tissues were harvested $24 \mathrm{~h}$ after i.v injection of the cathepsin-activatable NIRF probe. Stomach tissues were then scanned with a planar Odyssey near-infrared reader at $800 \mathrm{~nm}$ for visualization of the NIRF probe. The NIRF-probe signal is shown in green. Bright field imaging (red colour) to show the background was performed at $680 \mathrm{~nm}$. IHC analysis of probe-injected (C) and non-injected (F) mice indicates that cathepsins are expressed in both animals stomach tissues.

\section{Proteases activate NIRF-activable probe in gastric cancer}

Cathepsins B, H, L, and S are activators of an established near infrared fluorescent (NIRF) probe that is based on fluorescence resonance energy transfer [13]. To investigate activation of the cathepsinactivable near infrared fluorescent (NIRF) probe (prosense-750) ex vivo, $150 \mu \mathrm{l}$ of prosense-750 was intravenously (I.V) injected into CEA-TAG mice bearing gastric cancer. Twenty-four hours after intravenous administration of the probe, the mouse was sacrificed, the stomach tissues were prepared and visualized on an Odyssey planar near-infrared scanner to detect the signal of the NIRF probe. Tissue sections displayed a strong signal (Figures $5 \mathrm{~A}$ and $5 \mathrm{~B}$ ) emitted by the cleavage of NIRF probe in stomach tumor. Normal parts of stomach mucosa in fundus and corpus region displayed unspecific background auto-fluorescence, which was also observed in non-injected control mice (Figures 5C-5F). Immunohistochemical staining for cathepsin B indicated that gastric tumors of both prosense-injected and the control (non-injected) littermates displayed strong signal (Figures 4C and 4F).

\section{Discussion}

High grade dysplasia and early gastric cancer are often missed during conventional endoscopic examination of the upper gastrointestinal tract $[4,21]$. Candidate biomarker molecules that can be visualized by specific probes, labelled with fluorophores, are extremely needed to indicate high grade dysplasia and onset of early gastric cancer in endoscopic imaging [22]. The pattern of expression of such biomarker molecules in normal tissues, early tumor and late metastasis varies according to their respective functions in cells [23]. Some of such wellstudied cellular molecules are involved in angiogenesis, extracellular matrix degradation and dissemination of tumor cells [12,24]. Among potentially important candidate biomarker molecules for imaging early onset of gastric cancer are cathepsin proteases and matrix metalloprotinases [25]. Cathepsins are lysosomal cysteine proteolytic enzymes involved in several cellular functions including protein turnover, proliferation, invasion and metastasis whereas matrix metalloprotinases are zinc-dependent endopeptidases that are engaged in large variety of cellular physiology and pathology including tissue remodeling and metastasis $[19,26]$. They are frequently dysregulated in cancer and other cancer-associated cells. Apart from these, cathepsins and MMPs have been reported to be promising tools for early detection of tumors in various organs $[5,11,27]$. In our study, the expression pattern of cathepsins and MMPs was determined in human and murine gastric cancer cell lines. Relatively elevated expression of cathepsins B, $\mathrm{H}$ and MMP2 proteinases was observed in both human and murine gastric cancer cell lines. To corroborate tumour-specificity of the expression of these proteins we carried out qPCR and detected elevated level of the enzymes in tumor tissues but not in adjacent normal mucosa of murine stomach. Both immunohistochemical study and qPCR indicated relatively elevated expression of the target proteins, indicating potential application of the proteins in molecular imaging. This is line with several findings in other tumour types reported at different times [5,21]. In one of these studies, illustrating critical importance of the application of proteases in the diagnosis of curable precursor lesions and early-stage cancer, Eser and his colleagues were able to differentiate between normal pancreatic tissue and low-grade pancreatic intraepithelial neoplasia (mPanINs), high grade mPanINs and early-stage pancreatic ductal adenocarcinoma [5]. Furthermore, immunohistochemistry with antibodies specific to cathepsins and MMPs indicated that cathepsins B and $\mathrm{H}$ and MMP2 and MMP3 were strongly expressed in the CEA-TAG mice stomach tumor tissue than adjacent normal mucosa. Taken together, these findings indicate that proteases are potentially involved in gastric carcinogenesis and could be potential biomarkers for early detection of gastric cancer in mouse models. Thus, these proteins represent an attractive target for tumor imaging at molecular level. Moreover, these findings also point out that CEA-TAG mice will be an elegant mouse model for investigation of early gastric cancer and open a window of opportunity for monitoring and treatment evaluation of gastric cancer.

In contemporary era of molecular imaging using biological targets, enzymes and proteins provide functional visualization, which may help achieve the clinical goals of early detection and characterization of cancerous tissue in stomach [28]. Near infrared fluorescent imaging has demonstrated significant promise and advantage over other modalities in that it visualizes deeper tissues and minimizes tissue autofluorescence $[6,7,9]$. Among others, cathepsin-activatable and matrix metalloproteinase (MMP)-activatable probes have gained greater attention in near infrared fluorescent imaging technologies $[9,29]$. In this study, as cathepsins B and H and MMP2 are highly expressed in both human and murine gastric cancer, a cathepain-activatable probe was used to test a proof-of-principle for feasibility to detect gastric cancer by fluorescent imaging in mouse models. After 24 hours of the probe injection we performed ex-vivo fluorescent imaging. We were able to show that gastric tumors display a stronger fluorescent signal than adjacent normal mucosa. Therefore, detection of elevated cathepsin or MMP activity in regions of gastric dysplasia or cancer is potentially an effective tool for molecular probe based detection of gastric tumors by endoscopic fluorescent imaging of the stomach. The principle is that the injected probes were optically inactive and undetectable before they interact with their respective cysteine proteases. They are activated upon 
Citation: Labisso WL, Seifu D (2017) Application of Proteases as Molecular Imaging Tools for Early Gastric Cancer Detection: In Vitro and Ex Vivo Investigation. J Mol Biomark Diagn 8: 366. doi: 10.4172/2155-9929.1000366

protease cleavage permitting emission of NIRF of sites of protease upregulation and probe activation. The probe activation is visualized after $24 \mathrm{hrs}$ of injection and even it is tissue specific in that it is expressed only tumour tissues where these enzymes are highly expressed but not in normal mucosa. Only marginal signal or background is observed in the normal mucosa of the mice. Taken together, our results indicate that proteases are powerful molecular tools for early detection of pre-neoplastic and neoplastic tissues in stomach. However, further studies are necessary to answer the following questions: 1) is it possible to detect precancerous gastric lesions and early gastric cancer by an endoscopic imaging of protease activity in vivo? 2) Is it possible to monitor therapeutic response by imaging of cathepsin $\mathrm{B}$ and $\mathrm{H}$ or MMP2 in vivo? Finally, cathepsin B and H or MMP2 proteases are perhaps important molecules to mark the boundary between normal tissue and tumorigenic tissue, enhancing the efficacy of gastric cancer surgery.

\section{Conclusion}

Cathepsins and MMPs are potential biomarkers molecules for detection of prenoplastic and neoplastic lesions and early gastric cancer in mouse models, revealing the future applicability of the biomarkers in tumor imaging and therapeutic monitoring. However, further investigation is required to confirm the application of these enzymes for monitoring therapeutic responses and their prognostic values in gastric cancer in vivo.

\section{Ethics Approval and Consent to Participate}

All animal studies were conducted meeting the requirements of the European guidelines for the care and use of laboratory animals and were approved by the local animal ethics authorities in Bayerische region, Germany and research ethics committee of pathology department, Addis Ababa University, Ethiopia.

\section{Competing Interests}

We declare no conflict of interest.

\section{Funding and Author Contributions}

No funding was obtained from anywhere for this study. 1Designed and conducted the experiment; wrote the manuscript. 2Critically reviewed the manuscript, assisted in data analysis.

\section{Acknowledgements}

The authors are grateful to Prof. Dr. Saur for allowing the animal experiments to be conducted in his lab. We are also thankful to KAAD and pathology department Addis Ababa University.

\section{References}

1. Voutilainen ME, Juhola MT (2005) Evaluation of the diagnostic accuracy of gastroscopy to detect gastric tumours: Clinicopathological features and prognosis of patients with gastric cancer missed on endoscopy. Eur J Gastroenterol Hepatol 17: 1345-1349.

2. Milne AN, Sitarz R, Carvalho R, Carneiro F, Offerhaus GJ (2007) Early onse gastric cancer: On the road to unraveling gastric carcinogenesis. Curr Mol Med 7: 15-28.

3. Suvakovic Z, Bramble MG, Jones R, Wilson C, Idle N, et al. (1997) Improving the detection rate of early gastric cancer requires more than open access gastroscopy: A five-year study. Gut 41: 308-313.

4. Amin A, Gilmour H, Graham L, Paterson-Brown S, Terrace J, et al. (2002) Gastric adenocarcinoma missed at endoscopy. J R Coll Surg Edinb 47: 681-684.

5. Eser S, Messera M, Eser P, Von Werder A, Seidler B et al. (2011) In vivo diagnosis of murine pancreatic intraepithelial neoplasia and early-stage pancreatic cancer by molecular imaging. Proc Natl Acad Sci USA 108: 9945-9950.
6. Weissleder R (2006) Molecular imaging in cancer. Science 312: 1168-1171.

7. Ding S, Blue RE, Chen Y, Scull B, Kay LP, et al. (2012) Molecular Imaging of gastric neoplasia with near infrared fluorescent (NIRF) activatable probes. Mol Imaging 11: 507-515.

8. Zhang H, Morgan D, Cecil G, Burkholder A, Ramocki N, et al. (2008) Biochromoendoscopy: molecular imaging with capsule endoscopy for detection of adenomas of the GI tract. Gastrointest Endosc 68: 520-527.

9. Hilderbrand SA, Weissleder R (2010) Near-infrared fluorescence: application to in vivo molecular imaging. Curr Opin Chem Biol 14: 71-79.

10. Deryugina EI, Quigley JP (2006) Matrix metalloproteinases and tumor metastasis. Cancer Metastasis Rev 25: 9-34.

11. Duffy MJ (1996) Proteases as prognostic markers in cancer. Clin Cancer Res 2: 613-618.

12. Murphy G, Nagase $H$ (2008) Progress in matrix metalloproteinase research. Mol Aspects Med 29: 290-308.

13. Mohamed MM, Sloane BF (2006) Cysteine cathepsins: Multifunctional enzymes in cancer. Nat Rev Cancer 6: 764-775.

14. Gocheva V, Joyce JA (2007) Cysteine cathepsins and the cutting edge of cancer invasion. Cell Cycle 6: 60-64.

15. Murphy G (2008) The ADAMs: Signalling scissors in the tumour microenvironment. Nat Rev Cancer 8: 929-941.

16. Turk B (2004) Targeting proteases: Successes, failures and future prospects. Nat Rev Drug Discov 5: 785-799.

17. Nelson AR, Fingleton B, Rothenberg ML, Matrisian LM (2000) Matrix metalloproteinases: Biologic activity and clinical implications. J Clin Oncol 18 1135-1149.

18. Foda HD, Zucker S (2001) Matrix metalloproteinases in cancer invasion, metastasis and angiogenesis. Drug Discov Today 6: 478-482.

19. Thompson JA, Eades-Perner AM, Ditter M, Muller WJ, Zimmermann W (1997) Expression of transgenic carcinoembryonic antigen (CEA) in tumor-prone mice: An animal model for CEA-directed tumor immunotherapy. Int $\mathrm{J}$ Cancer 72: 197-202.

20. Cavallo-Medved D, Rudy D, Blum G, Bogyo M, Caglic D, et al. (2009) Livecell imaging demonstrates extracellular matrix degradation in association with active cathepsin B in caveolae of endothelial cells during tube formation. Exp Cell Res 315: 1234-1246.

21. Von Burstin J, Eser S, Seidler B, Meining A, Bajbouj M, et al. (2008) Highly sensitive detection of early-stage pancreatic cancer by multimodal nearinfrared molecular imaging in living mice. Int J Cancer 123: 2138-2147.

22. Leodolter A, Kulig M, Brasch $H$, Meyer-Sabellek W, Willich SN, et al. (2006) A meta-analysis comparing eradication, healing and relapse rates in patients with Helicobacter pylori-associated gastric or duodenal ulcer. Aliment Pharm Ther 15: 1949-1958.

23. Gounaris E, Tung HC, Restaino C, Maehr R, Kohler R, et al. (2008) Live imaging of cysteine cathepsin activity reveals dynamics of focal inflammation, angiogenesis, and polyp growth. PLoS One 3: e2916.

24. Weissleder R, Ntziachristos $V$ (2003) Shedding light onto live molecular targets Nat Med 9: 123-128.

25. Egeblad M, Werb Z (2002) New functions for the matrix metalloproteinases in cancer progression. Nat Rev Cancer 2: 161-174.

26. Keppler D, Sloane BF (1996) Cathepsin B: Multiple enzyme forms from a single gene and their relation to cancer. Enzyme Protein 49: 94-105.

27. Weissleder R, Tung CH, Mahmood U, Bogdanov A Jr (1999) In vivo imaging of tumors with protease-activated near-infrared fluorescent probes. Nat Biotechnol 17: 375-378.

28. Herszenyi L, Plebani M, Carraro P, De Paoli M, Roveroni G, et al. (2000) Proteases in gastrointestinal neoplastic diseases. Clin Chim Acta 291: 171-187.

29. Metildi CA, Kaushal S, Snyder CS, Hoffman RM, Bouvet M (2013) Fluorescenceguided surgery of human colon cancer increases complete resection resulting in cures in an orthotopic nude mouse model. J Surg Res 179: 87-93. 